\title{
An Equation of Mordell
}

\section{By Andrew Bremner}

Abstract. All integer solutions of the Diophantine equation $6 y^{2}=(x+1)\left(x^{2}-x+6\right)$ are found.

1. Mordell [1] asks if all the integer solutions of the Diophantine equation $6 y^{2}=(x+1)\left(x^{2}-x+6\right)$ are given by $x=-1,0,2,7,15$ and 74 . It is shown that there are precisely seven integer solutions, the seventh with $x=767$.

Consideration of factorization gives, tor some integers $a, b$,

$$
\begin{aligned}
& \left\{\begin{array} { l } 
{ x ^ { 2 } - x + 6 = 6 b ^ { 2 } } \\
{ x + 1 = a ^ { 2 } }
\end{array} \text { or } \left\{\begin{array}{l}
x^{2}-x+6=3 b^{2} \\
x+1=2 a^{2}
\end{array}\right.\right. \text { or } \\
& \left\{\begin{array} { l } 
{ x ^ { 2 } - x + 6 = 2 b ^ { 2 } } \\
{ x + 1 = 3 a ^ { 2 } }
\end{array} \text { or } \left\{\begin{array}{l}
x^{2}-x+6=b^{2} \\
x+1=6 a^{2}
\end{array}\right.\right.
\end{aligned}
$$

and the latter case is impossible modulo 3. We thus obtain, on eliminating $x$, the three quartic equations,
(i) $a^{4}-3 a^{2}+8=6 b^{2}$,
(ii) $4 a^{4}-6 a^{2}+8=3 b^{2}$,
(iii) $9 a^{4}-9 a^{2}+8=2 b^{2}$.

The standard technique in dealing with equations of this type is to factorize in the appropriate quadratic extension of the integers, which here is $Z[(1+\sqrt{-23}) / 2]$, to obtain a finite set of equations of the form,

$$
a^{2}=f(v, w), \quad 1=g(v, w),
$$

where $f, g$ are homogeneous quadratic forms.

We need to know some details of the quadratic field $Q(\sqrt{-23})$. The class-number of the ring of integers is 3 ; and we denote the ideal factorizations of 2 and 3 by (2) $=\mathfrak{p}_{2} \overline{\mathfrak{p}}_{2},(3)=\mathfrak{p}_{3} \overline{\mathfrak{p}}_{3}$ where a bar denotes conjugacy, and $\mathfrak{p}_{2} \mathfrak{p}_{3}=((1+\sqrt{-23}) / 2)$.

Thus in Eq. (i), $\left(2 a^{2}-3\right)^{2}+23=24 b^{2}$ implies the ideal equation

$$
\left(\frac{2 a^{2}-3 \pm \sqrt{-23}}{2}\right)=q \mathfrak{b}^{2} \text { where } q \bar{q}=(6) \text { and } \mathfrak{b} \text { is some integral ideal. }
$$

There are essentially two possibilities, $q=\mu_{2} p_{3}$ and $q=\bar{p}_{2} p_{3}$. In the former instance, $\mathfrak{b}$ is principal, and in the latter, $\mathbf{b} \overline{\mathfrak{p}}_{2}$ is principal.

Since $p_{3} \bar{p}_{2}^{-1}=((1+\sqrt{-23}) / 4)$ we have, respectively,

Received October 29, 1974.

AMS (MOS) subject classifications (1970). Primary $10 \mathrm{~B} 10$.

Key words and phrases. Diophantine equation.

Copyright $\odot 1975$, American Mathematical Society 
and

$$
\pm\left(\frac{2 a^{2}-3 \pm \sqrt{-23}}{2}\right)=\left(\frac{1+\sqrt{-23}}{2}\right)\left(\frac{u+v \sqrt{-23}}{2}\right)^{2}
$$

$$
\pm\left(\frac{2 a^{2}-3 \pm \sqrt{-23}}{2}\right)=\left(\frac{1+\sqrt{-23}}{4}\right)\left(\frac{u+v \sqrt{-23}}{2}\right)^{2}
$$

for some integers $u, v$ satisfying $u \equiv v \bmod 2$. Thus we have, respectively,

and

$$
\left\{\begin{aligned}
-\left(2 a^{2}-3\right) & =\frac{u^{2}-46 u v-23 v^{2}}{4} \\
1 & =\frac{u^{2}+2 u v-23 v^{2}}{4}
\end{aligned}\right.
$$

$$
\left\{\begin{aligned}
\left(2 a^{2}-3\right) & =\frac{u^{2}-46 u v-23 v^{2}}{8} \\
-1 & =\frac{u^{2}+2 u v-23 v^{2}}{8}
\end{aligned}\right.
$$

where the signs in each equation have been determined by a congruence modulo 3 .

In the former case, putting $u+v=2 w$, we obtain

$$
\text { I: }\left\{\begin{aligned}
a^{2} & =w^{2}+12 w v-12 v^{2} \\
1 & =w^{2}-6 v^{2}
\end{aligned}\right.
$$

In the latter case, $u^{2}+2 u v+v^{2} \equiv 0 \bmod 8$, so $u+v=4 w$ say; then

$$
\text { II: }\left\{\begin{aligned}
a^{2} & =6 v^{2}-12 v w-2 w^{2} \\
1 & =3 v^{2}-2 w^{2} .
\end{aligned}\right.
$$

In similar manner (ii) gives rise to

and (iii) to the three pairs

$$
\text { III: }\left\{\begin{aligned}
a^{2} & =-2 w^{2}+12 w v-6 v^{2} \\
1 & =4 w^{2}-3 v^{2}
\end{aligned}\right.
$$

and

$$
\text { IV: }\left\{\begin{array} { c } 
{ a ^ { 2 } = v ( 9 v + 1 6 w ) } \\
{ 1 = 9 v ^ { 2 } - 8 w ^ { 2 } }
\end{array} \quad \text { V: } \left\{\begin{array}{r}
a^{2}=v(v+8 w) \\
1=v^{2}-18 w^{2}
\end{array}\right.\right.
$$

$$
\mathrm{VI}:\left\{\begin{aligned}
a^{2} & =32 w(v-9 w) \\
1 & =v^{2}-288 w^{2}
\end{aligned}\right.
$$

Of these six pairs of equations, V and VI may be treated by simple descent arguments. For instance, in VI, we have that $w=m^{2}$ or $2 m^{2}$ after change of sign if necessary: so it suffices to determine all integer solutions of the equations $1=v^{2}-18 m^{4}$ and $1=$ $v^{2}-72 m^{4}$, respectively. This is readily achieved by means of a classical descent argument; but we can quote Ljunggren [2] to say that the only integer solutions of the former are $( \pm r, \pm m)=(1,0)$ and $(17,1)$, and of the latter $( \pm r, \pm m)=(1,0)$. These give the solutions $a=0$ and $a=16$ of Eq. (iii) whence solutions $x=-1,767$ of the original equation. 
Each of the four remaining pairs of equations represents the intersection of two quadrics in three-dimensional space; the method of solution, as exploited by Cassels [3] , is to consider the singular elements in the pencil of the quadrics. Such singular quadrics are given by $f-\lambda g$, where $\operatorname{det}(f-\lambda g)=0$ : that is, a linear combination of $f$ and $g$ which is a perfect square. In general, of course, $\lambda$ is a quadratic irrational. We can thus rewrite each pair of equations in the form $a^{2}-\mu L(v, w)^{2}=\lambda$ for some $\mu \in$ $Q(\lambda)$, where $L(v, w)$ is a homogeneous linear form with coefficients in $Q(\lambda)$. We now work over $\mathrm{Q}(\delta)$ where $\delta^{2}=\mu$ and equate $(a+L \delta)$ and $(a-L \delta)$ as ideals, to two ideal factors of $\lambda$ in $\mathbf{Q}(\delta)$, noting that the two factors must be conjugate over $\mathbf{Q}(\mu)$. All the ideals are principal, so using the appropriate arithmetical details of the field $Q(\delta)$, we can equate coefficients of elements of an integer base; in particular, it is clear that the coefficient of $\delta^{2}$ in $a+L \delta$ is zero, and the resulting equation is completely solved by congruence considerations.

As an illustration, consider Eq. II. The singular quadrics in this pencil are obtained by taking a linear combination which is a perfect square: so let $3(2+\lambda) v^{2}-12 w v-2(1+\lambda) w^{2}$ be a perfect square. Then $36=-6(1+\lambda)(2+\lambda)$ or $\lambda^{2}+3 \lambda+8=0$. Taking $\lambda=(-3-\sqrt{-23}) / 2$ we obtain

$$
a^{2}-(1+\sqrt{-23})\left[w-\frac{1-\sqrt{-23}}{4} v\right]^{2}=\frac{3+\sqrt{-23}}{2},
$$

and accordingly work in $\mathbf{Q}(\delta)$ where $\delta^{2}=1+\sqrt{-23}$. We need some arithmetical details of this field; certainly $\tau=\left(\delta^{3}-2 \delta^{2}+2 \delta+4\right) / 8$ is an algebraic integer, since $\tau^{2}-\tau((1-\sqrt{-23}) / 2)+1=0$. The discriminant of $R=Z\left[1, \delta, \delta^{2} / 2, \tau\right]$ is $2^{3} \cdot 3 \cdot 23^{2}$, whence $R$ is indeed the ring of integers of the field (for 23 certainly ramifies, so $23^{2}$ divides the discriminant, and Stickelberger's criterion says that the discriminant is congruent to 0 or 1 modulo 4 ). It is also readily calculated by standard techniques that $\tau$ is a fundamental unit for the field, and that we have the factorization, (2) $=q_{2}\left(q_{2}^{\prime}\right)^{2}$, where $q_{2}=\mathfrak{p}_{2},\left(q_{2}^{\prime}\right)^{2}=\bar{p}_{2}$, with $\mathfrak{p}_{2}=(2,(1+\sqrt{-23}) / 2)$, $\bar{p}_{2}=(2,(1-\sqrt{-23}) / 2)$.

The equation now becomes in terms of ideals,

$$
\left(a+\delta\left(w-\frac{v}{2}\right)+\frac{\delta^{3}}{4} v\right)\left(a-\delta\left(w-\frac{v}{2}\right)-\frac{\delta^{3}}{4} v\right)=\left(\mathfrak{q}_{2}^{\prime}\right)^{6}
$$

and since the two ideals on the left are conjugate over $\mathbf{Z}[(1+\sqrt{-23}) / 2]$ we must have

$$
\left(a+\delta\left(w-\frac{v}{2}\right)+\frac{\delta^{3}}{4} v\right)=\left(\mathfrak{q}_{2}^{\prime}\right)^{3}=\left(\frac{\delta^{3}-6 \delta+16}{4}\right) .
$$

Because there are no nontrivial roots of unity in $\mathbf{Q}(\delta)$ we now obtain $a+$ $\delta(w-v / 2)+\left(\delta^{3} / 4\right) v= \pm\left(\left(\delta^{3}-6 \delta+16\right) / 4\right) \tau^{n}$ for some integer $n$. This exponential equation is solved by first comparing coefficients of $\delta^{2}$, using the fact that $\tau^{5} \equiv-1$ mod 7; a congruence modulo a suitable power of 7 then shows that the only solutions are given by $n=0$ or -3 . These give $a=4$ as solution of (i), and $x=15$ as a solution of the original equation.

The complete details of the proof are to appear in my $\mathrm{Ph} . \mathrm{D}$. Thesis. I gratefully thank Professors Swinnerton-Dyer and Cassels for their advice and encouragement. 
Emmanuel College

Cambridge, England

1. L. J. MORDELL, Diophantine Equations, Pure and Appl. Math., vol. 30, Academic Press, London and New York, 1969, p. 259. MR 40 \#2600.

2. W. LJUNGGREN, "Einige Eigenschaften der Einheiten reeller quadratischer und reinbiquadratischer Zahlkorper," Oslo Vid.-Akad. Skrifter, v. 1, 1936, no. 12.

3. J. W. S. CASSELS, "Integral points on certain elliptic curves," Proc. London Math. Soc. (3), v. 14A, 1965, pp. 55-57. MR 31 \#2200. 